\title{
LORENTZ DETUNING OF SUPERCONDUCTING CAVITIES WITH UNBALANCED FIELD PROFILES*
}

\author{
J. R. Delayen", Thomas Jefferson National Accelerator Facility, Newport News, VA 23606, USA
}

\section{Abstract}

Tests on the prototype SNS medium beta cryomodule showed a strong correlation between the flatness of the field profile and the Lorentz detuning coefficient (both static and dynamic). We present an analytical model for the enhancement of the Lorentz detuning as a function of the flatness of the field profile resulting from a spread of the frequencies of the individual cells of the cavity.

\section{INTRODUCTION}

Lorentz detuning of superconducting cavities is the change of frequency associated with the deformation of the geometry induced by the radiation pressure of the electromagnetic field. In all practical applications, this detuning is proportional to the square of the amplitude of the electromagnetic field. Measurements on the SNS prototype cryomodule have shown large variations of the Lorentz coefficient -by more than a factor of 2 - between supposedly identical cavities [1,2]. There seemed to be, however, a correlation between the flatness of the field profile and the Lorentz coefficient. In this paper we calculate analytically and numerically the change of the Lorentz detuning coefficient associated with the flatness of the field profile.

\section{TWO-CELL CAVITY MODEL}

We represent a 2-cell cavity as two coupled harmonic oscillators of frequencies $\omega_{1}$ and $\omega_{2}$ with a coupling strength $k \omega_{0}^{2}$. This coupled system is represented by

$$
\left\{\begin{array}{c}
\ddot{x}_{1}+\omega_{1}^{2} x_{1}=k \omega_{0}^{2} x_{2} \\
\ddot{x}_{2}+\omega_{2}^{2} x_{2}=k \omega_{0}^{2} x_{1}
\end{array} .\right.
$$

The two eigenvalues (frequencies of the resonating modes) and eigenvectors (amplitudes of the fields in the cells) are

$$
\begin{aligned}
& \omega_{a, b}^{2}=\frac{\omega_{1}^{2}+\omega_{2}^{2} \pm\left[\left(\omega_{1}^{2}-\omega_{2}^{2}\right)^{2}+4 \omega_{0}^{4} k^{2}\right]^{1 / 2}}{2}, \\
& \frac{x_{1}}{x_{2}}=\frac{2 k \omega_{0}^{2}}{\omega_{1}^{2}-\omega_{2}^{2} \mp\left[\left(\omega_{1}^{2}-\omega_{2}^{2}\right)^{2}+4 \omega_{0}^{4} k^{2}\right]^{1 / 2}} .
\end{aligned}
$$

Cavities are usually operated in the $\pi$-mode where the amplitudes have opposite signs in the two cells. This implies choosing the upper sign in the above equations. If we assume that the frequencies of the two cells have

\footnotetext{
* Work supported by the U.S. Department of Energy under contract DE-AC05-00-OR22725

\#delayen@jlab.org
}

different values such that $\omega_{1}^{2}=\omega_{0}^{2}(1+\alpha)$ and $\omega_{2}^{2}=\omega_{0}^{2}(1-\alpha)$, then the frequency of the " $\pi$-mode" is $\omega_{\pi}^{2}=\omega_{0}^{2}\left[1+\left(\alpha^{2}+k^{2}\right)^{1 / 2}\right]$ and the ratio of the amplitudes in the two cells is $\frac{x_{1}}{x_{2}}=\frac{k}{\alpha-\left(\alpha^{2}+k^{2}\right)^{1 / 2}}$.

Often, the operating gradient of an accelerating cavity is defined not in terms of the actual voltage provided, which is not directly measurable in the absence of a beam, but through the intermediary of the energy content. The relationship between energy content and gradient is obtained and defined for a well-balanced structure. Thus, a comparison of the Lorentz detuning between a balanced and unbalanced structure will be made first at constant energy content i.e. we will assume that $x_{1}^{2}+x_{2}^{2}=2$. With this assumption, the amplitudes in the two cells of an unbalanced structure are

$$
\begin{aligned}
& x_{1}=\frac{k}{\left(\alpha^{2}+k^{2}\right)^{1 / 4}\left[\left(\alpha^{2}+k^{2}\right)^{1 / 2}-\alpha\right]^{1 / 2}}, \\
& x_{2}=\frac{\alpha-\left(\alpha^{2}+k^{2}\right)^{1 / 2}}{\left(\alpha^{2}+k^{2}\right)^{1 / 4}\left[\left(\alpha^{2}+k^{2}\right)^{1 / 2}-\alpha\right]^{1 / 2}} .
\end{aligned}
$$

We now introduce Lorentz detuning by assuming that the frequencies of the two cells change by amounts proportional to the square of their amplitudes:

$$
\omega_{1}^{2}=\omega_{0}^{2}\left(1+\alpha-\beta x_{1}^{2}\right), \quad \omega_{2}^{2}=\omega_{0}^{2}\left(1-\alpha-\beta x_{2}^{2}\right) .
$$

We assume that the changes in frequency induced by Lorentz detuning are small enough that the amplitudes are still given by Eq. (1.3). From Eq. (1.1), and using the upper sign, the frequency of the " $\pi$-mode" is

$$
\omega_{\pi}^{2}=\omega_{0}^{2}\left\{1-\beta+\left[\alpha^{2}\left(1-\frac{\beta}{\left(\alpha^{2}+k^{2}\right)^{1 / 2}}\right)^{2}+k^{2}\right]^{1 / 2}\right\},
$$

and the ratio of Lorentz-induced frequency shift with and without asymmetry between the two cells is

$$
\begin{aligned}
\frac{\Delta \omega_{\pi}^{2}(\alpha \neq 0)}{\Delta \omega_{\pi}^{2}(\alpha=0)} & =\frac{\beta-\left[\alpha^{2}\left(1-\frac{\beta}{\left(\alpha^{2}+k^{2}\right)^{1 / 2}}\right)^{2}+k^{2}\right]^{1 / 2}+\left(\alpha^{2}+k^{2}\right)^{1 / 2}}{\beta} \\
& =1+\frac{\alpha^{2}}{\alpha^{2}+k^{2}} \frac{2-\frac{\beta}{\left(\alpha^{2}+k^{2}\right)^{1 / 2}}}{1+\left[1-\frac{\alpha^{2} \beta}{\left(\alpha^{2}+k^{2}\right)^{3 / 2}}\left(2-\frac{\beta}{\left(\alpha^{2}+k^{2}\right)^{1 / 2}}\right)\right]^{1 / 2}} .
\end{aligned}
$$


Thus the enhancement due to asymmetry $(\alpha \neq 0)$ should always be less than 2. In fact, if we make the realistic assumption that the Lorentz detuning at the operating field is much less than the cell-to-cell coupling $(\beta \ll k)$ the enhancement simplifies to

$$
\frac{\Delta \omega_{\pi}^{2}(\alpha \neq 0)}{\Delta \omega_{\pi}^{2}(\alpha=0)}=1+\frac{\alpha^{2}}{\alpha^{2}+k^{2}}=1+\frac{\varepsilon^{2}}{1+\varepsilon^{2}},
$$

where $\varepsilon=\alpha / k$

These results apply to identical "assumed" gradients; actually they are at identical energy content and the actual gradient will be less than the "assumed" gradient. With the above notations and with $y_{i}=\left|x_{i}\right|$, at an energy content $y_{1}^{2}+y_{2}^{2}=2$, the energy gain in a balanced structure will be $y_{1}+y_{2}=2$. In an unbalanced structure, the real energy gain at the same energy content will be

$$
y_{1}+y_{2}=\frac{1-\varepsilon+\left(1+\varepsilon^{2}\right)^{1 / 2}}{\left(1+\varepsilon^{2}\right)^{1 / 4}\left[\left(1+\varepsilon^{2}\right)^{1 / 2}-\varepsilon\right]^{1 / 2}}<2
$$

In the case of extreme field unbalance, only one cell will be energized and the actual energy gain will be $\sqrt{2}$ instead of the assumed 2.

When comparing the Lorentz detunings at identical real gradients, the result of Eq. (1.4) needs to be multiplied by

$$
\frac{4}{\left(y_{1}+y_{2}\right)^{2}}=2 \frac{\left(\alpha^{2}+k^{2}\right)^{1 / 2}}{1+\left(\alpha^{2}+k^{2}\right)^{1 / 2}},
$$

and the detuning enhancement at constant real gradient is

$$
\frac{\Delta \omega_{\pi}^{2}(\alpha \neq 0)}{\Delta \omega_{\pi}^{2}(\alpha=0)}=\frac{2}{1+\left(1+\varepsilon^{2}\right)^{1 / 2}} \frac{1+2 \varepsilon^{2}}{\left(1+\varepsilon^{2}\right)^{1 / 2}}
$$

The field flatness can be defined simply in a 2-cell structure as

$$
\Phi=\frac{\left|y_{1}-y_{2}\right|}{\frac{y_{1}+y_{2}}{2}}=2 \frac{1+\varepsilon-\left(1+\varepsilon^{2}\right)^{1 / 2}}{1-\varepsilon+\left(1+\varepsilon^{2}\right)^{1 / 2}} .
$$

The Lorentz detuning enhancement constant energy content and constant energy gain given by Eqs. (1.4) and (1.5), respectively, are shown in Fig. 1 as function of the field flatness defined by Eq. (1.6).

When the cells are almost identical (i.e. when their frequency difference is much less than the cell-to-cell coupling), the enhancements at constant energy content and constant energy gain are $1+\Phi^{2}$ and $1+1.25 \Phi^{2}$, respectively. In the case of extreme difference between the two cells the enhancement at constant energy content is 2 , while, at constant energy gain, it is 4 .

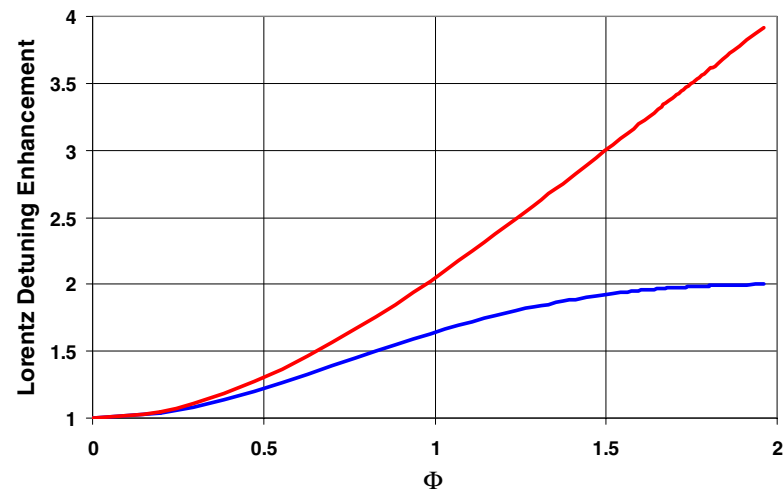

Figure 1: Lorentz detuning enhancement for a 2-cell structure as a function of field flatness between the two cells. Blue curve is at constant energy content; red curve is at constant energy gain.

\section{MULTI-CELL CAVITIES}

In the case of cavities with a large number $(N)$ of different cells, the modes frequencies and amplitudes, and the Lorentz detuning coefficient cannot be obtained analytically and a numerical analysis is required. Additionally there are several ways of defining the field flatness, and there is no one-to-one relationship between any of those and the Lorentz detuning enhancement.

One way of defining the field flatness is

$$
\Phi_{1}=\frac{\max \left(y_{i}\right)-\min \left(y_{i}\right)}{\langle y\rangle} .
$$

Another way is through a standard deviation

$$
\Phi_{2}=\left(\frac{N}{N-1}\right)^{1 / 2}\left[\sum \frac{\left(y_{i}-\langle y\rangle\right)^{2}}{\langle y\rangle^{2}}\right]^{1 / 2} .
$$

It can be noted that, for a 2-cell structure, these two definitions are identical to each other and to that given by Eq. (1.6); and that, in the case of extreme field unbalance where only on cell is energized, we have $\Phi_{1}=\Phi_{2}=N$.

We have analyzed numerically a large number of multicell structures with random deviations of the frequencies of each individual cells from an average. This amounts to finding the eigenvalues and eigenvectors of tridiagonal $N \times N$ matrices to obtain the frequencies without Lorentz detuning, and then solving the problem again where the frequency of each cell has been changed further by an amount proportional to the square of the amplitude in that cell. The change in frequency of the " $\pi$-mode" is then compared to the one that would occur in a similar structure where all the cells are identical.

Results for 6-cell structures are shown in the Figures 26. The same numerical analysis was applied to 2-cell structures and the analytical results of section 2 were recovered. 


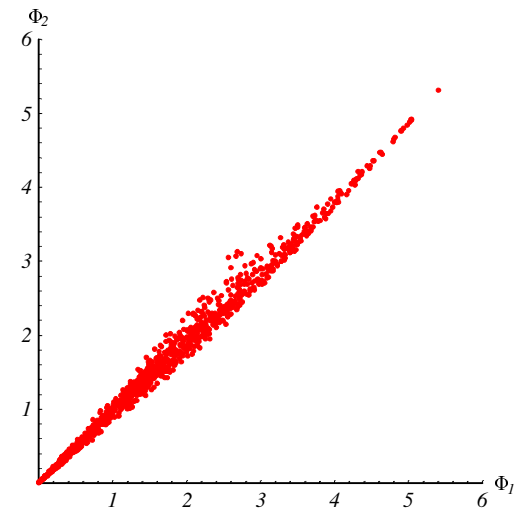

Figure 2: Correlation between the field flatness defined by Eqs. (1.7) and (1.8).

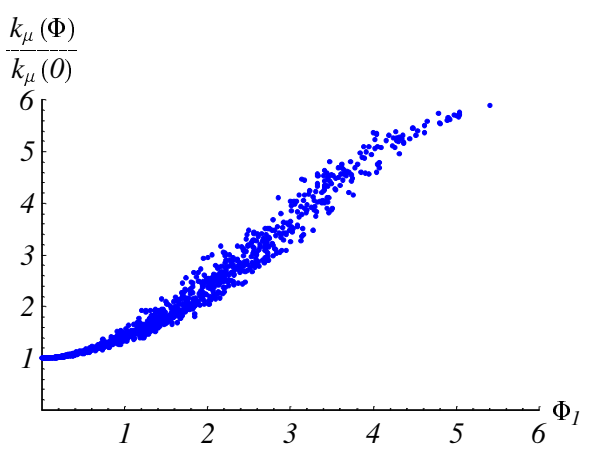

Figure 3: Lorentz detuning enhancement at constant energy content for field flatness defined by Eq. (1.7).

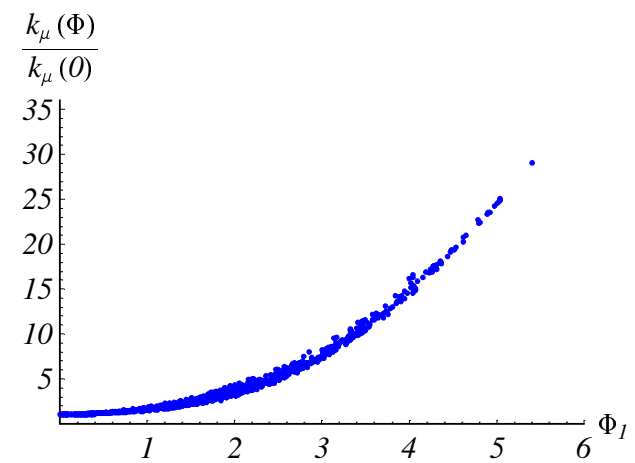

Figure 4: Lorentz detuning enhancement at constant energy gain for field flatness defined by Eq. (1.7).

\section{DISCUSSION}

During the measurements on the SNS prototype cryomodule, Lorentz detuning coefficients of the three cavities were measured. Variations of more than a factor of 2 were observed and seemed to be correlated with the flatness of the field profile, which, when measured at room temperature, was up to $70 \%$ according to definition given by Eq. (1.7). The present analysis supports this correlation but cannot explain such a large difference, if

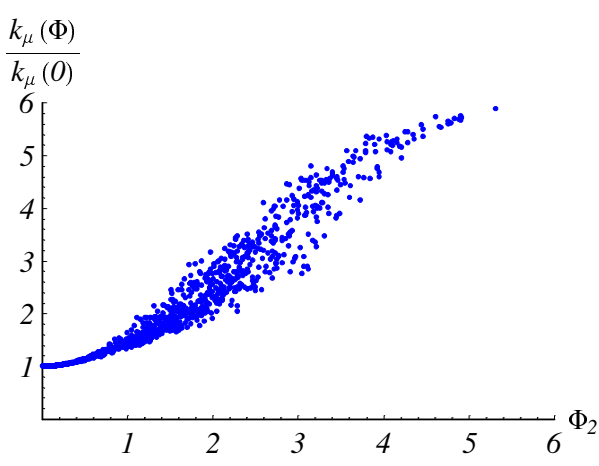

Figure 5: Lorentz detuning enhancement at constant energy content for field flatness defined by Eq. (1.8).

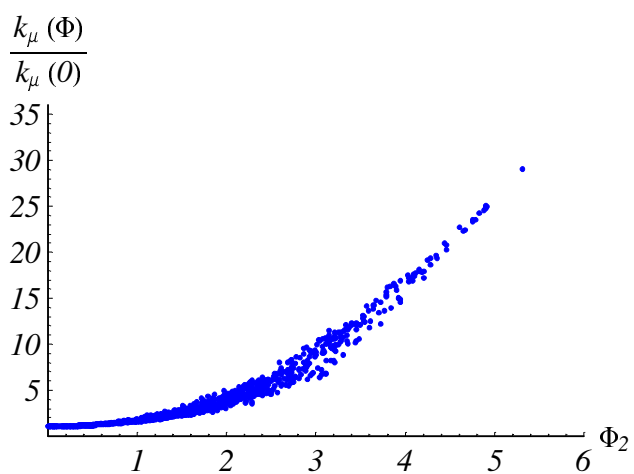

Figure 6: Lorentz detuning enhancement at constant energy gain for field flatness defined by Eq. (1.8).

the field flatness remained the same during cooldown, and other factors may have contributed.

Analysis of a large number of cavities with random perturbation of the cell frequencies show similar correlation between the two definitions of the field flatness and the Lorentz coefficient enhancement. Definition (1.7) seems to be better correlated with the detuning enhancement for large flatness coefficients, while, for low values of the flatness coefficient, there is a better correlation with the flatness defined by Eq. (1.8).

\section{REFERENCES}

[1] Jean Delayen, Ed Daly, Kirk Davis, Steve Smee, "Frequency Measurements on the Prototype SNS Medium- $\beta$ Cryomodule Under Pulsed and CW Operation," JLab Tech Note 02-049.

[2] I. Campisi, G. Ciovati, G. K. Davis, J. R. Delayen, M. Drury, L. W. Funk, J. Mammosser, T. Powers, J. Preble, C. E. Reece, C. H. Rode, M. Stirbet, H. Wang, T. Witlatch, K. M. Wilson, M. Wiseman, "The SNS Prototype Cryomodule: Testing and Performance," these proceedings RPAB065. 